\title{
Adaptation of the Residual Signal for Filter Failure Detection in Scenarios with Multiple Filter Types
}

\author{
Sima Barzegar, Marc Ruiz, and Luis Velasco" \\ Optical Communications Group (GCO), Universitat Politècnica de Catalunya (UPC), Barcelona, Spain \\ *e-mail:lvelasco@ac.upc.edu
}

\begin{abstract}
To monitor the optical spectra of outgoing links, Optical Spectrum Analyzers (OSA) can deployed in the optical nodes acquiring the optical spectra of outgoing links and analyzing optical signals to detect those soft-failures that affect the shape of the spectrum, like Filter Shift and Filter Tightening. In this paper, we rely on the residualbased approach spectra analysis that uses a set of residual signals calculated by subtracting the measured signal by the OSA from an expected version of the signal. In this paper, we assume a network scenario, where nodes with optical filters of different characteristics (e.g., $2^{\text {nd }}, 3^{\text {rd }}$, and $4^{\text {th }}$ order Gaussian) are installed. In this scenario, the possibility of generating complete data set for machine learning training purposes that include every combination of filter and failure is difficult to achieve. Instead, we propose an adaptation mechanism that transform the expected effects of every filter type thus, emulating single filter-type networks. Exhaustive experiments are carried out to show the performance of the proposed adaptation under filter failures.
\end{abstract}

Keywords: optical spectrum monitoring, monitoring and data analytics, machine learning for optical networks

\section{INTRODUCTION}

Network performance monitoring is a key enabler for failure identification and localization, which can greatly bring down both, the repair time and operational cost of optical networks. Many research efforts have been dedicated to develop failure localization techniques for hard failures, i.e., unexpected events that suddenly interrupt the established connections. Nonetheless, although some works can be found in the literature focused on the identification and localization of soft failures, i.e., events that progressively degrade the quality of transmission (QoT), this topic remains rather unexplored. Owing to the fact that soft failures might eventually evolve to hard failures, it is of paramount importance not only to detect them a priori before connections disruption, but also to localize their cause in order to take proper action, e.g., finding a restoration path. Such monitoring data can be used by data analytics applications, especially those based on machine learning (ML) [1].

Recently, the authors in [2] proposed several solutions to monitor the performance of lightpaths at the transponders side to verify their proper operation, as well as to detect bit-error-rate (BER) degradations. The authors studied several soft-failure causes affecting signal QoT, such as Filter Shift (FS) and Filter Tightening $(F T)$, and proposed algorithms to detect and identify the most probable failure. Some of these failures happen in the optical switching intermediate nodes, so monitoring the signal solely at the egress node (or even ingress) does not allow their localization. Hence, monitoring techniques to evaluate QoT in-line are required.

As the abovementioned failures noticeably affect the optical spectrum of the lightpaths, Optical Spectrum Analyzers (OSA) can be used to monitor the spectrum along the transmission line aiming at detecting and localizing that type of failures. Practically speaking, the realization of such solutions become possible with the emergence of a new generation of compact cost-effective OSAs with sub-GHz resolution in the form of optical components [3] allowing real-time monitoring of the optical spectrum of the lightpaths.

Considering the optical spectrum of a lightpath, when a signal is properly configured, its central frequency should be around the center of the assigned frequency slot to avoid filtering effects, and it should be symmetrical with respect to its central frequency. The authors in [4], presented several descriptive features to characterize the optical spectrum of a lightpath. Such features were used to train a set of ML algorithms to detect and identify failures. The most common filtering related failures are FS and FT; the optical spectrum becomes asymmetrical in the case of FS, and its edges get noticeably rounded in the case of FT. These irregularities allow distinguishing optical spectra suffering from such failures from the properly configured ones.

Authors in [5] extend the work in [4] and study different feature-based approaches that use optical spectrum features for classification, as well as the residual-based approach, in which the received signal is pre-processed using a theoretically-calculated expected signal. Note that one single filter type was considered in [4] and [5], which limits the deployment of ML approaches in real operator networks that usually consist of equipment from different vendors. The most straightforward solution to overcome this limitation is to have different models being trained upon various types of filters that might be available in the network. Nonetheless, it makes the training phase very complex and data-hungry. Yet, it will not be easy to comprehend the sequence of filters $a$ priori and the responses of a slightly non-identical filters in the network might not be well detected, necessitating even more combination of models to have an appropriate generic model. In this paper, we present an enhanced version of such approach with the capability of getting adapted to new filter types in the network. The application of out-of-field model training and in-field adaptation [6] leads to a robust, yet feasible, solution for 
networks with heterogeneous filtering; just one type of filter will be used for ML training and adaptation of the optical spectrum acquired from OSA that have passed through several type of different filters are developed.

\section{RESIDUAL-BASED APPROACH}

Optical spectrum acquired by OSAs is collected and can be used for failure detection. An example of a 30GBaud QPSK modulated optical spectrum acquired by an OSA is shown in Fig. 1a. In general, QPSK and 16QAM -modulated optical signals present, once filtered, a flat spectral region around the central frequency, sharp edges, and a round region between the edges and the central one. In contrast, the impact of FS and FT on the properly operating lightpaths makes that some or all these features change, as presented in Fig. 1b-c, where the impact of filter cascading after crossing several wavelength selective switches (WSS) is also depicted.

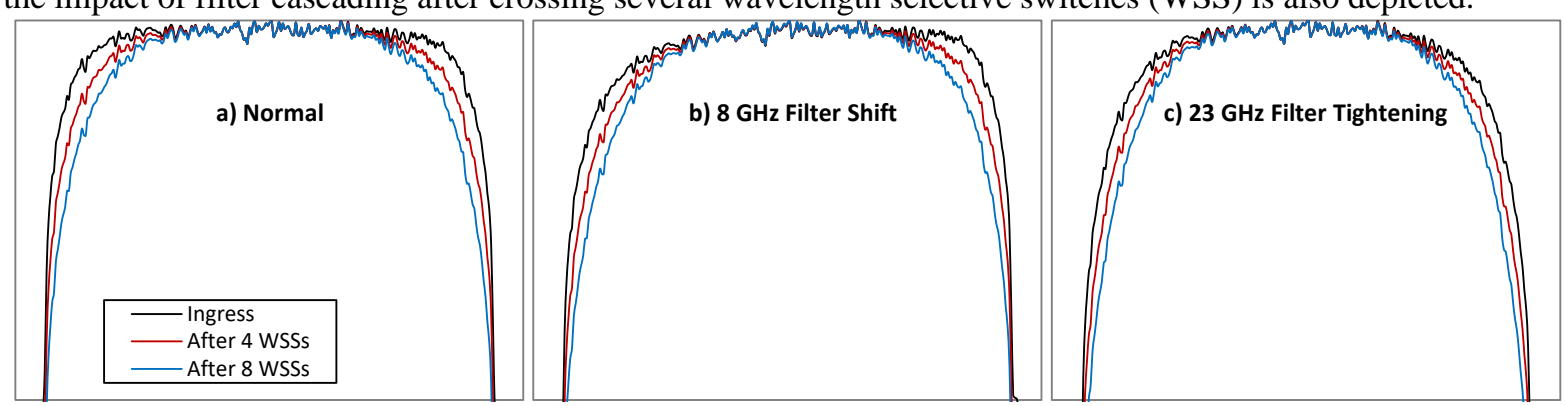

Fig. 1. The shape of the optical spectrum for a normal signal (a), a signal experiencing FS (b), and experiencing FT (c).

The residual-based approach lies in pre-processing the acquired optical spectrum by comparing it to the one that would be expected after passing the same number of filters than the signal. This comparison produces a residual representing the differential deformation that it is used as input for a classifier that detects soft-failures (Fig. 2).

Two modules are required to compute the residual signal (Fig. 3a): $i$ ) the Expected Signal Calculator (ESC) (Fig. 3b) and ii) the Residual Computation and Adaptation (Fig. 3c). The ESC module generates a theoretically-calculated spectrum emulating a properly operating lightpath. The aim of ESC is to

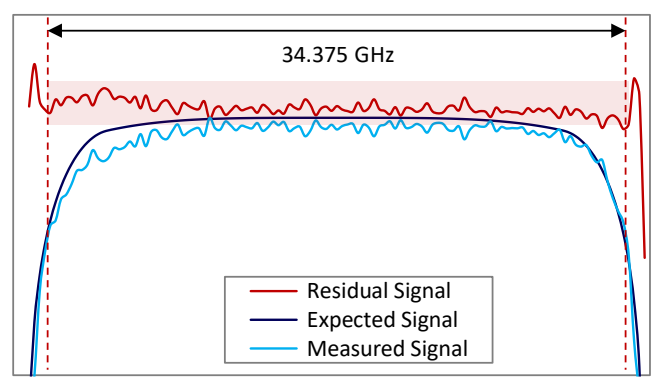

Fig. 2. Residual signal calculation. synthetically reproduce an averaged noise-free version of the measured signal. Then, the residual signal is easily obtained by subtracting the acquired signal by the OSA from the signal generated by the ESC module. However, further elaboration on the residual signal is required to make it suitable for decision-making and training the classifiers. The residual signals can ultimately be used to train SVM-based classifiers to detect (i.e., decide whether a signal is normal or affected by a failure) and identify filter failures (i.e., decide between FT and FS).

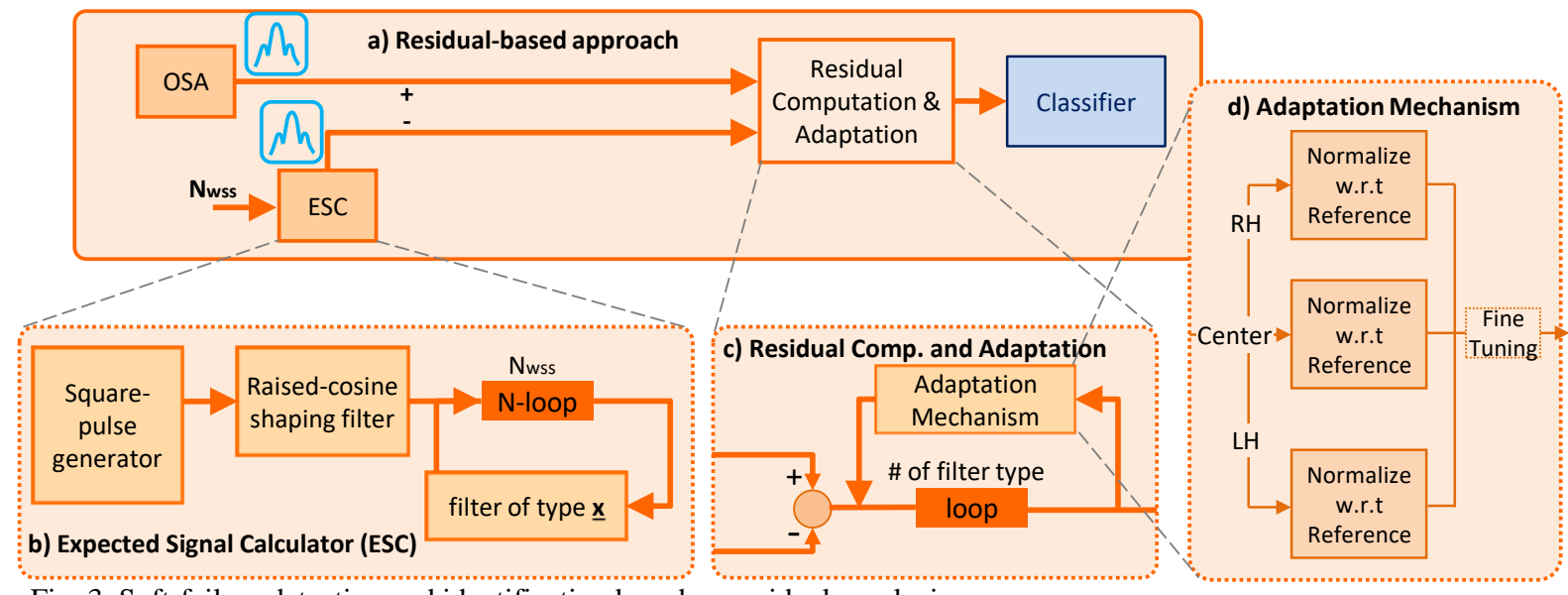

Fig. 3. Soft-failure detection and identification based on residuals analysis

Ultimately, a single classifier trained with the measurements based on a reference filter type, can be used for optical spectra experiencing filtering effects from different types of filter. The classifier was implemented as a SVM that produces a diagnosis which consists of: $i$ ) a predicted class among 'Normal' and 'FilterFailure'; and ii) a subset of relevant signal points for the predicted class. In the case that a filter failure is detected, another classifier is used to predict whether the failure is due to FS or to FT. 


\section{SIGNAL ADAPTATION}

The ESC module performs signal adaptation by considering the specific filters that the signal has passed through (see three filter transfer functions in Fig. 4a). In addition, the residual computation module normalizes and adapts the residuals for the signal under analysis. The calculated residual is normalized with respect to the mean value of the central part of the residual, so the mean becomes 0 . This normalization approach is operational when the same type of filter is considered for ML training and operation of the ML algorithm, whereas it produces distorted results in the presence of other filter types. In view of this, we propose an adaptation procedure (Fig. 3d) that consists in dividing the residual signal in three segments (see Fig. 4b) and apply different normalization methods to every segment, reflecting the filter characteristics; the normalization reference of every segment is obtained by applying linear regression to the un-normalized version of the residual signal obtained for that segment. Then, the residual computation and adaptation module receives the signal, as well as the linear regression coefficients modeling three different normalization references that consider the filter characteristics.

For this stage, the number of adaptation mechanism loop equals to the number of filter types that the lightpath has passed through. By subtracting every segment of the un-normalized residuals from the corresponding normalization reference, a filter type-agnostic residual signal is obtained. Note that, as the amount of filter cascading effect depends on the transfer function of the filter, there might be an undesirable deviation in the residual signals

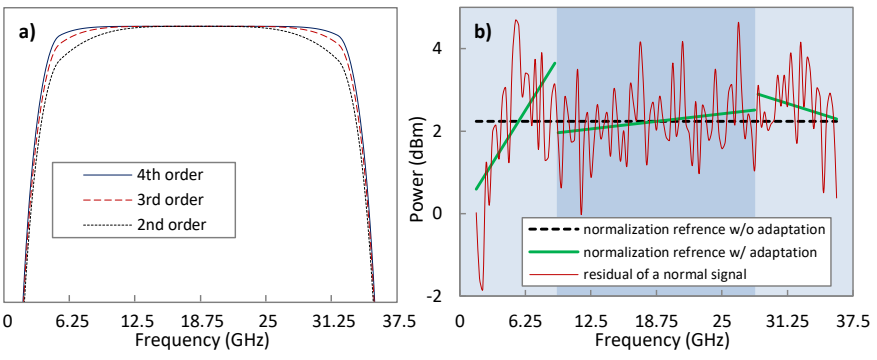

Fig. 4. a) Three filter types and b) 4th order Gaussian normalization.

when the lightpath traverses different filter types; this deviation is compensated in the fine-tuning step. The amount of deviation can be computed locally assuming that the mean value of the residual remains zero when the signal is in proper operation mode.

\section{ILLUSTRATIVE RESULTS}

In this section, we demonstrate how the proposed adaptation mechanism enables the residual-based approach to be applied to optical spectrum of a signal after passing through different types of filters.

For the experiments, we configured a VPIPhotonics scenario (Fig. 5) where a $100 \mathrm{~Gb} / \mathrm{s}$ DP-QPSK modulated signal was emulated. After the transmitter, the optical signal passes through eight optical nodes (from N1 to N8); after every span, an optical amplifier compensates for the accumulated attenuation of the fiber. Every optical node consists of two WSSs, each one modeled as a single optical filter with a $2^{\text {nd }}$ order

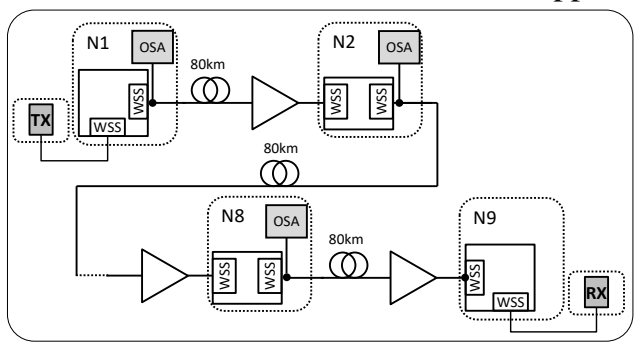

Fig. 5. VPI setup

Gaussian transfer function for the training phase; filters bandwidth is set to $37.5 \mathrm{GHz}$, leaving $7.5 \mathrm{GHz}$ as a guard band for the lightpath. Finally, the optical signal ends in a coherent receiver that compensates for the impairments introduced throughout the transmission. In addition, OSAs are placed after every optical node to acquire the optical spectrum of every optical link.

The efficiency of the proposed adaptation method is illustrated in Fig. 6. The residual signals of a lightpath passing through three different types of filters with Gaussian transfer function of order 2,3, and 4 are illustrated in Fig. 6a. Normalization shifts the residuals so its mean to be 0 (Fig. 6b). Note that the differences among residuals are clearly seen at the edges, whereas they are virtually identical in the central part before and after normalization. Adaptation focus on compensating the effects of the different filters and the results are clearly visible at the edges (Fig. 6c); note that the most relevant parts of the residuals to detect filter-related soft-failures are that of the edges. As shown, even though the signals pass through different types of filters, they result in an identical residual signal, removing the filter-dependent characteristics of the residual signal.

Aiming at emulating failure scenarios, we modified the characteristics of the $2^{\text {nd }}$ WSS of every node in the setup; its bandwidth and central frequency were modified to model FT and FS failures, respectively. A large dataset of failures was collected by inducing failures of magnitude in the range [1-8] $\mathrm{GHz}$ for FS and in the range [1-15] GHz for FT. We configured optical filters to be $2^{\text {nd }}$ order Gaussian for training and re-configured them to become $3^{\text {rd }}$ and $4^{\text {th }}$ order Gaussian for testing, where the same failure scenarios were simulated.

We looked firstly at the benefits of applying the adaptation mechanism for identifying the normal cases. We found that accuracy is very poor $(<20 \%)$ when no adaptation is applied and becomes perfect with residual adaptation. Next, we looked at the benefits of applying residual adaptation for detecting failures. Three cases were studied: i) $2^{\text {nd }}$ order for both out-of-field training and in-field testing; note that no adaptation is needed, ii) $3^{\text {rd }}$ order and ii) $4^{\text {th }}$ order, in which $2^{\text {nd }}$ order filters were used for training and $3^{\text {rd }}$ and $4^{\text {th }}$ order, respectively w/ 
adaptation were used for testing. The results are reported in Fig. 7, where Fig. 7(a-b) show the average node accuracy of identifying FS and FT, respectively, for failures in all 8 nodes and varying levels of failure magnitudes. The accuracy is promising for all the cases under study, even though it degrades for very small magnitudes in which the spectrum looks like normal cases; in fact, failure detection is $100 \%$ in all cases being the failure identification step the cause of the reduced accuracy. To highlight the impact of cascaded nodes, Fig. 7c presents the average accuracy for FS and FT with respect to the node where the failure occurs; failure magnitudes of range [1-4] GHz for FS and [4-7] GHz for FT were considered. As shown, the accuracy drops at the very last nodes as a result of accumulated filter cascading effects makes very challenging to distinguish between different cases.

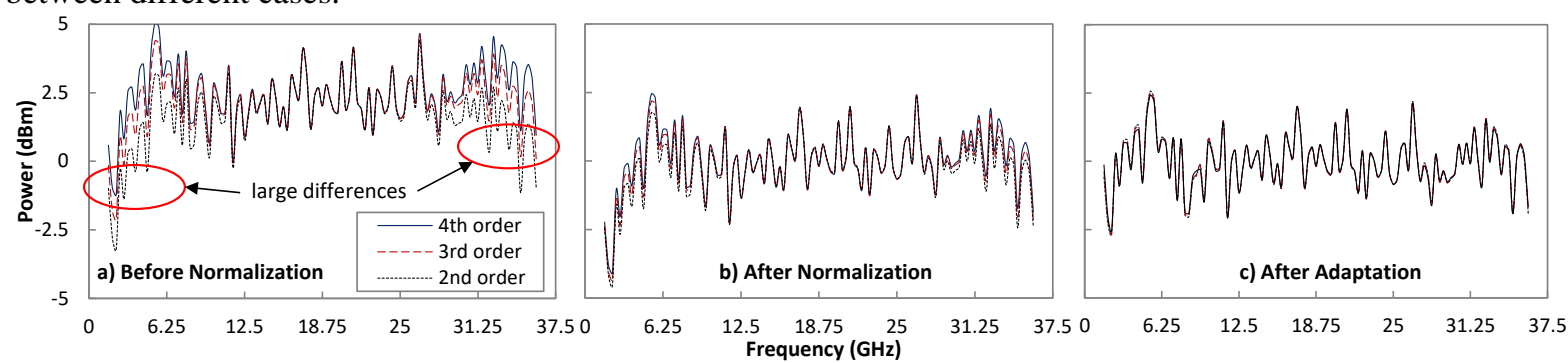

Fig. 6. a) Un-normalized residual, b) normalized w/o adaptation, and c) normalized with adaptation.
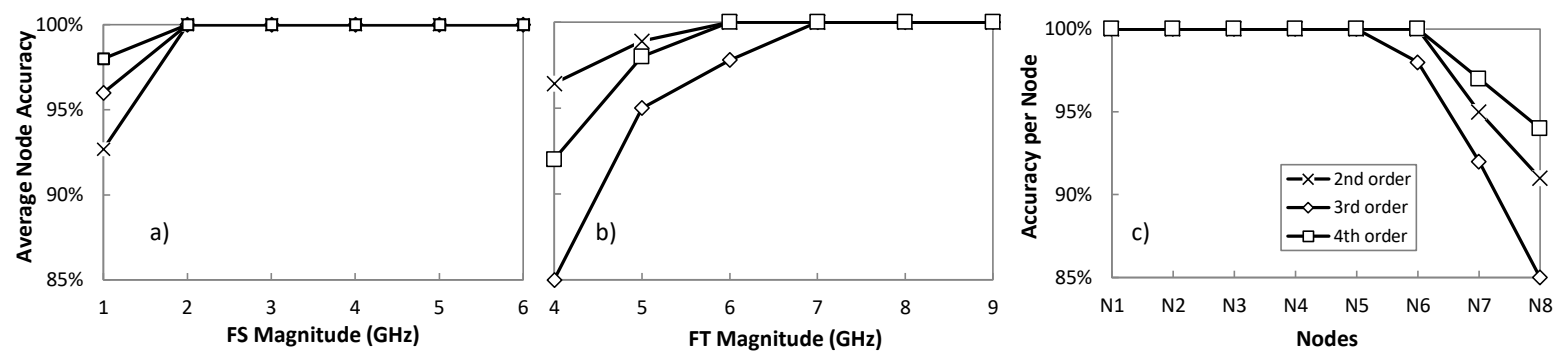

Fig. 7. Average node accuracy w.r.t failure magnitudes for a) FS and b) FT. c) Accuracy per node w.r.t the cascaded nodes.

The efficiency of the adaptation algorithm for transmission systems with two different filter types was evaluated. To this end, we modified the above-described setup to have $2^{\text {nd }}$ order Gaussian filters in the first 4 nodes and $4^{\text {th }}$ order Gaussian filters in the last 4 ones. Failure detection accuracy is $100 \%$ while failure identification shows perfect accuracy for magnitudes above some values. Specifically, the minimum failure magnitude to be detected with $100 \%$ accuracy is 5 and $7 \mathrm{GHz}$, for FS and FT, respectively, just a bit higher than in the case of one single filter type. These results validate the performance of our residual adaptation method.

\section{CONCLUSIONS}

A use case of out-of-field training with in-field model adaptation was studied as a scalable option to obtain accurate models and algorithms for filter-related failure detection and classification in optical networks with heterogeneous transmission and switching devices. The out-of-field ML training option is studied through a lightpath failure detection and identification use case, where the spectra acquired by OSAs installed in intermediate nodes was analyzed for filter-related failures detection and identification. The residual-based approach was selected due to its potential characteristics to get adapted to different types of filter because of its dependency on the synthetic behavior of the filter responses.

\section{ACKNOWLEDGEMENTS}

This work was partially supported by the EC through the METRO-HAUL project (G.A. $\mathrm{n}^{\circ}$ 761727), from the AEI/FEDER TWINS project (TEC2017-90097-R), and from the Catalan ICREA Institution.

\section{REFERENCES}

[1] D. Rafique and L. Velasco, "Machine Learning for Optical Network Automation: Overview, Architecture and Applications," (Invited Tutorial) IEEE/OSA J. of Optical Communications and Networking (JOCN), vol. 10, 2018.

[2] A. P. Vela et al., "BER degradation detection and failure identification in elastic optical networks," IEEE/OSA Journal of Lightwave Technology (JLT), vol. 35, pp. 4595-4604, 2017.

[3] Flexgrid High Resolution Optical Channel Monitor (OCM) [On-line] www.finisar.com, accessed June 2018.

[4] A. P. Vela et al., "Soft Failure Localization during Commissioning Testing and Lightpath Operation [Invited]," IEEE/OSA Journal of Optical Communications and Networking (JOCN), vol. 10, pp. A27-A36, 2018.

[5] B. Shariati, M. Ruiz, J. Comellas, and L. Velasco, "Learning from the Optical Spectrum: Failure Detection and Identification [Invited],” IEEE/OSA Journal of Lightwave Technology (JLT), vol. 37, pp. 433-440, 2019.

[6] L. Velasco et al., "A Learning Life-Cycle to Speed-up Autonomic Optical Transmission and Networking Adoption," IEEE/OSA Journal of Optical Communications and Networking, vol. 11, pp. 226-237, 2019. 